\title{
Adaptation of the Feedforward Postural Response to Repeated Continuous Postural Perturbations
}

\author{
Ashleigh Kennedy ${ }^{1,2}$, Nicoleta Bugnariu ${ }^{3}$, Arnaud Guevel $^{2}$, Heidi Sveistrup ${ }^{1,4}$ \\ ${ }^{1}$ School of Human Kinetics, Faculty of Health Sciences, University of Ottawa, Ottawa, Canada; ${ }^{2}$ Laboratory "Motricité, Interactions, \\ Performance" (EA 4334), University of Nantes, Nantes, France; ${ }^{3}$ University of North Texas Health Science Centre, Physical Therapy, \\ Fort Worth, USA; ${ }^{4}$ School of Rehabilitation Sciences, Faculty of Health Sciences, University of Ottawa, Ottawa, Canada. \\ Email: apurn097@uottawa.ca
}

Received January $9^{\text {th }}, 2013$; revised February $10^{\text {th }}, 2013$; accepted February $22^{\text {nd }}, 2013$

\begin{abstract}
We examined the adaptation of the postural response to repeated predictable platform oscillations. Our main goals were to determine whether the short-term changes that occurred during a minute long continuous postural perturbation trial were maintained in subsequent trials and to determine how many trials were required before participants fully adapted to the postural task. Ten participants performed ten minute-long postural trials on a platform that oscillated at $0.25 \mathrm{~Hz}$ before increasing to $0.50 \mathrm{~Hz}$ half way through each trial. Postural muscle onset latencies, burst amplitudes, and anterior posterior displacements of the center of pressure (COP) and center of mass (COM) were calculated for the last five cycles performed in each trial at $0.50 \mathrm{~Hz}$. The postural strategy evolved in two phases: 1) immediate decrease in COP displacement; 2) earlier activation of the postural muscles with smaller muscle burst amplitudes. After seven trials the postural response remained consistent.
\end{abstract}

Keywords: Adaptation; Postural Control; Continual Perturbation

\section{Introduction}

Postural stability is maintained during externally initiated perturbations through modifications of the postural muscle activity and center of pressure (COP) displacement [1-4]. These changes allow effective control of postural stability in novel conditions; however, they may be initially inefficient, requiring a high level of tibialis anterior (TA) and soleus (Sol) muscle activity [5]. With further experience short-term adaptation occurs, allowing the participants to tailor their postural response to meet the requirements of the environmental and internal variables $[6,7]$.

In discrete postural perturbations experience provides participants with performance feedback resulting in activation of postural muscles earlier in advance of the perturbation and at smaller muscle burst amplitudes [8]. Postural adaptation also occurs during continuous, sinusoidal oscillations of the platform [5,9-11]. In this type of recurrent, continual perturbation the postural response evolves rapidly to meet the environmental requirements. In fact, within only 3 to 5 sinusoidal platform oscillations young healthy adults transition from a reactive to an anticipatory postural response $[10,11]$.

It is clear that short-term changes occur during both discrete and continuous postural perturbations; however it has not been determined whether the rapid changes that occur during one postural trial are maintained in the steady state postural response of subsequent trials. To address this question we examined how postural muscle activity, center of mass and center of pressure displacement changed over the steady state of 10 distinct continuous postural trials. The secondary aim of this study was to determine how many trials were required for the participant to reach a stable and consistent postural response. We hypothesized that it would take several trials for the postural response to reach this point and that once the response was stabilized; the ankle muscles would be consistently activated earlier in anticipation of the postural perturbation with smaller muscle burst amplitudes [8]. Since the COM is a priority variable in dynamic postural control [12], we hypothesized that it would be maintained while the COP displacement adapted to meet the environmental and task requirements.

\section{Methods}

\subsection{Participants}

Ten (five men, five women) young healthy subjects (aged 19 - 27; men height $180.8 \pm 6.3 \mathrm{~cm}$, weight $77.8 \pm$ 
$10.4 \mathrm{~kg}$; women height $166.7 \pm 17.7 \mathrm{~cm}$, weight $57.6 \pm$ $9.4 \mathrm{~kg}$ ) with no neurological impairment or history of serious injury that prevented them from participating in sport for more than 6 months gave informed consent to participate in this study. Elite athletes (collegiate level or higher) and dancers (participation more than four times/ week) were excluded from the sample. The experimental procedures were approved by the ethics board at the University of Ottawa and were performed in accordance with the Tri-Council Policy Statement [13].

\subsection{Measurement Devices}

Data from seven VICON cameras (Vicon Peak, Oxford, UK) and thirty-six retro-reflective markers, placed on anatomical landmarks and on four corners of the force plate, were used to estimate the position of the center of mass $(\mathrm{COM})$ and the platform during the postural trials. A movable platform instrumented with a Kistler force plate (Type 9286, Kistler Instrument Corp, New York, USA) was used to record the ground reaction forces at $500 \mathrm{~Hz}$ during the postural trials (Nexus, Vicon Peak, Oxford, UK). Kinematic data were recorded at $200 \mathrm{~Hz}$.

Surface electrodes (Myomonitor Wireless Delsys EMG system, Boston, USA) recorded electromyographical (EMG) activity from the gastrocnemius medialis (GM), biceps femoris (BF), tibialis anterior (TA) and rectus femoris (RF) in accordance with SENIAM recommendations [14]. A ground electrode was placed on the patella. The EMG signals were pre-amplified, sampled at 1000 $\mathrm{Hz}$ and full wave rectified.

\subsection{Procedure}

Ten postural trials, each consisting of $\sim 60$ seconds of continuous, sinusoidal oscillations of the platform in the anterior/posterior direction, were performed. Fifteen seconds of rest was afforded between each postural trial. During the trials participants stood barefoot with their eyes open and feet shoulder width apart on the platform as it oscillated $20 \mathrm{~cm}$ in the anterior-posterior plane (for further details see Bugnariu and Sveistrup 2006). In each postural trial, $8 \pm 2$ oscillations were performed at 0.25 $\mathrm{Hz}$ before the frequency of oscillation increased to 0.50 $\mathrm{Hz}$ for another $10 \pm 2$ cycles.

\subsection{Data Analysis}

The VICON Plug-in Gait biomechanical model, combined with anthropometric measurements and kinematic data, was used to estimate the forward and backward displacement of the center of mass (COM). The anterior/ posterior movement of the platform was subtracted from the COM data in order to provide a true representation of COM displacement relative to the base of support. Ground reaction forces were used to estimate the maxi- mum anterior/posterior COP displacement.

Muscle burst onsets were determined using a two standard deviation threshold derived from the quiet stance period that occurred before every trial. Once the thresholds were identified (BioProc version 3.06, D.G.E. Robertson), muscle onset latencies were visually coded relative to the start of the forward or backward platform translations. Muscle onsets occurring in anticipation of the platform movement were coded as negative. Dynamic responses had to be recorded in at least $25 \%$ of the directionally specific oscillations in order to be included in calculations of group muscle onset latencies.

The COM and COP displacement, muscle onset latency and amplitude of the TA, GM, RF and BF were analyzed for the first (T1), third (T3), fifth (T5), seventh (T7) and tenth (T10) trials. In each trial, the values for the last five cycles at $0.50 \mathrm{~Hz}$ were averaged [10]. Data were ensemble averaged across participants in each of the time periods and reported for forward and backward platform translations separately.

\subsection{Measurement Devices}

Repeated measures analyses of variance (ANOVA) tests were used to determine whether COP and COM displacement and muscle burst amplitudes adapted with experience following forward and backward oscillations. Posthoc analyses performed with Tukey corrections were used to determine the point at which no further adaptation occurred. Muscle onset latencies of the four postural muscles were not normally distributed so significant differences between trials were determined using nonparametric Wilcoxan sign-ranked tests.

\section{Results}

\subsection{COM and COP Displacement}

The COM displacement did not adapt over the 10 postural trials for the forwards $(\mathrm{F}(4,36)=0.654, \mathrm{p}>$ $0.05)$ or backwards $(\mathrm{F}(4,36)=0.345, \mathrm{p}>0.05)$ oscillations (data not shown). There was, however, a main effect of trial for the COP displacement in response to the forwards $(\mathrm{F}(4,36)=5.34, \mathrm{p}<0.05)$ and backwards $(\mathrm{F}(4,36)=4.02, \mathrm{p}<0.05)$ oscillations (Figure 1). Post-hoc analysis revealed that in forward direction the COP displacement decreased from $305.0 \pm 5.6 \mathrm{~mm}$ at $\mathrm{T} 1$ to $288.1 \pm 3.8 \mathrm{~mm}$ at $\mathrm{T} 3(\mathrm{p}=0.002)$. The COP displacement then stabilized, showing no significant adaptation between $\mathrm{T} 3$ and $\mathrm{T} 5(\mathrm{p}=0.387)$, T5 and $\mathrm{T} 7(\mathrm{p}=$ $0.775)$ or $\mathrm{T} 7$ and $\mathrm{T} 10(\mathrm{p}=0.625)$ (Figure 1). In the backwards direction the COP displacement decreased ( $\mathrm{F}$ $(4,36)=4.02, \mathrm{p}<0.05)$ from $296.0 \pm 5.5$ at $\mathrm{T} 1$ to 285.0 \pm 5.6 at $\mathrm{T} 3(\mathrm{p}=0.045)$ and again to $275.1 \pm 3.9$ at T5 (p $=0.043)$. After this point the COP displacement did not change further ( $\mathrm{T} 5$ to $\mathrm{T} 7 \mathrm{p}=0.318$ and $\mathrm{T} 7$ to $\mathrm{T} 10$ 


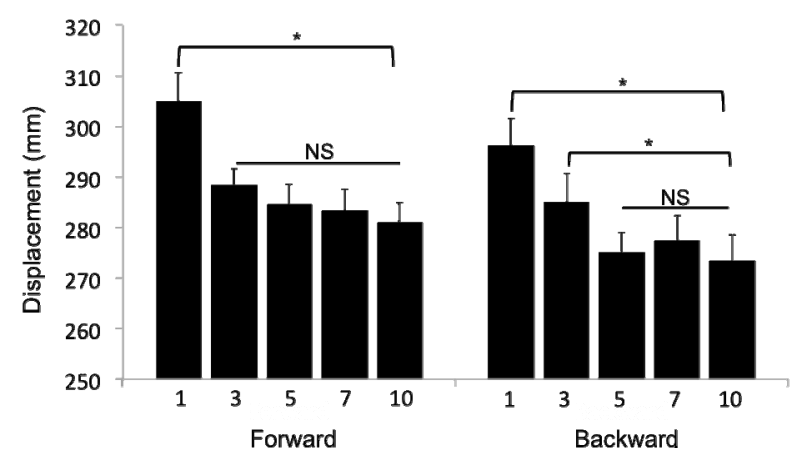

Figure 1. Center of pressure (COP) displacement during forward and backward translation of the platform. Following forward platform translation, the amplitude of COP displacement decreased significantly after the first trial (T1) with no further decrease through to the $10^{\text {th }}$ trial (T10). Following backwards translation, the COP decreased until the fifth trial (T5), with no additional decrease. Data displayed as mean $\pm \mathrm{SE}$. "indicates $\mathrm{p}<0.05$ and NS indicates no significant difference.

$\mathrm{p}=0.708)$

\subsection{Muscle Burst Amplitude}

The TA muscle burst amplitude adapted to the postural task over the first five trials $(\mathrm{F}(4,36)=20.268$, $\mathrm{p}<0.05)$ with a significant decrease between the amplitude found at $\mathrm{T} 1$ and $\mathrm{T} 3(\mathrm{p}=0.01)$ and between $\mathrm{T} 3$ and $\mathrm{T} 5(\mathrm{p}=$ $0.019)$. There was no further decrease between T5 and $\mathrm{T} 7(\mathrm{p}=0.053)$ or T7 and T10 $(\mathrm{p}=0.686)$. The muscle burst amplitude of the GM also adapted throughout the postural trials $(\mathrm{F}(4,36)=10.133, \mathrm{p}<0.05)$, decreasing significantly between $\mathrm{T} 1$ and $\mathrm{T} 3(\mathrm{p}=0.001)$. There was no further decrease between T3 and T5 ( $\mathrm{p}=0.299)$, T5 and T7 $(\mathrm{p}=0.21)$ or T7 and T10 $(\mathrm{p}=0.274)$. The RF muscle burst amplitudes, which were only assessed for trials T1, T5, T7 and T10, showed a significant main effect of adaptation $(\mathrm{F}(3,27)=3.294$, p < 0.05); however, post-hoc analysis did not reveal any specific locations of change throughout the 10 minute training period. The BF muscle amplitude, assessed for T1, T7 and T10, also showed a significant main effect of adaptation ( $\mathrm{F}(2$, 18), 3.805, $\mathrm{p}<0.05)$. Post-hoc analysis did not reveal any specific location of change between these time points.

\subsection{Muscle Onset Latency}

The TA muscle onset latency adapted to the postural task over 10 trials $\left(\mathrm{X}^{2}(4,10)=12.12, \mathrm{p}<0.05\right)$. There was no change between $\mathrm{T} 1$ and $\mathrm{T} 3(\mathrm{p}=0.385)$ or T3 and T5 $(\mathrm{p}=0.58)$; however, the TA was activated significantly earlier at T7 than it was at T5 $(\mathrm{p}=0.017)$ with no further change in the onset latency between T7 and T10 ( $\mathrm{p}=$ 0.35). The GM muscle onset latency also adapted to the postural task $\left(\mathrm{X}^{2}(4,10)=7.2, \mathrm{p}<0.05\right)$. There was no difference between the onset latency found at T1 and T3 $(\mathrm{p}=0.110)$ but the GM muscle was activated significantly earlier at T5 than it was at T3 $(p=0.041)$. After this point there was no further change in the GM muscle onset latency (T5 and T7 p $=0.929$, T7 and T10 p = 0.859) (Figure 2). Due to the infrequent muscle bursts, statistical analysis was only performed on the RF muscle onset latency at T1, T5, T7 and T10 and on the BF muscle onset latency at $\mathrm{T} 1, \mathrm{~T} 7$ and $\mathrm{T} 10$. Adaptation did not occur over these time points for either the $\mathrm{RF}\left(\mathrm{X}^{2}(3\right.$, $3)=7.4, p>0.05)$ or $\operatorname{BF}\left(X^{2}(4,2)=1.5, p>0.05\right)$.

\section{Discussion}

The aim of this study was to determine how the postural response adapted to meet the requirements of the motor task as well as to establish the point at which the motor task no longer changed with further experience. The hypothesis that the COM displacement would be maintained was based on previous research showing that the COM displacement is a performance variable that is preferentially controlled using muscle activity and individual joint motion in postural tasks $[5,15]$. The data from the present study confirmed our hypothesis, showing no change in the COM displacement over the 10 postural trials.

We also hypothesized that with experience the COP displacement and muscle activity would be progressively modified to maintain control of the COM in a more efficient way. Data from this study confirmed our hypothesis, showing a progressive adaption of the postural response throughout the first seven trials, after which point it no longer evolved. This adaptation process occurred in two phases beginning with a decrease in the COP displacement (Figure 1), followed by an earlier activation of the
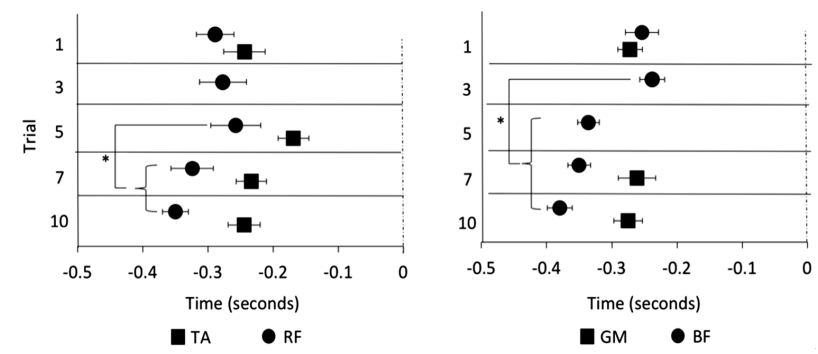

Figure 2. Muscle onset latencies of the tibialis anterior (TA) and rectus femoris (RF) coded relative to forward surface translations and the gastrocnemius medialis (GM) and biceps femoris (BF) coded relative to the backards surface translation. The TA and GM muscles were activated progressively earlier with experience. At some time points the RF and BF muscles were activated too infrequently to run statistical analyses (depicted by missing data); however, the onset latency did not change between the trials in which there were enough muscle bursts to assess statistically. Data displayed as mean $\pm \mathrm{SE}$. "indicates $\mathrm{p}<0.05$. 
postural muscles at a smaller muscle burst amplitude (Figure 2). This process is well described by the modified systems theory in which the participant initially constrains the body's degrees of freedom to improve motor control. Practice then optimizes the movement, improving the efficiency and adaptability of the task, allowing the participant to take advantage of the increased degrees of freedom [7].

At the start of the first phase the COP displacement was quiet large; however, the transition towards a more conservative postural response occurred rapidly, causing a significant decrease in the COP displacement between the first and third postural trials in both the forward and backwards directions. Similar to the temporal evolution of the postural kinetics found in previous research [16], the COP displacement measured in this study reached a stable level after 5 postural trials. After these initial changes the COP displacement no longer evolved with further experience, marking the end of the first phase of postural adaptation.

In the second phase of adaptation the postural response became more energy efficient. The postural muscles were recruited progressively earlier during this phase, reaching a stable level after 7 oscillations in the forward direction and 5 in backwards direction (Figure 2). Interestingly, these muscles were activated with smaller muscle burst amplitudes than were used in the first phase of adaptation (data not shown). Previous research has shown a similar decrease in the burst amplitude of the postural muscles as participants become more experienced with discrete [8] and continuous [5] postural perturbations, although no one has examined the evolution of the postural response from one continuous postural trial to the next. We postulate that these changes in muscle activity represent the optimization of the postural response to avoid fatigue and/or to reduce the expenditure of energy required for the task [7].

In conclusion, the data from this study demonstrated that young healthy participants transferred the experience gained in one postural trial to subsequent trials. With experience the COP displacement decreased and the postural muscles were activated earlier in anticipation of the perturbation with smaller muscle burst amplitudes. After seven, minute long trials, the anticipatory postural response no longer evolved with further experience. Experimental manipulations of factors influencing postural control, such as augmented feedback and/or fatigue, should be performed after this point to ensure that the postural changes found in the performance of the motor task are due to the experimental manipulation and not to the adaptation process.

\section{Acknowledgements}

The authors would like to acknowledge Coren Walters-
Stewart for her help with the data processing. This research was funded by the Ontario Graduate Scholarship in Science and technology. The authors have no direct conflict of interest that would influence the content of this study.

\section{REFERENCES}

[1] L. M. Nashner, "Fixed Patterns of Rapid Postural Responses among Leg Muscles during Stance," Experimental Brain Research, Vol. 30, No. 1, 1977, pp. 13-24. doi:10.1007/BF00237855

[2] F. B. Horak and L. M. Nashner, "Central Programming of Postural Movements: Adaptation to Altered Support-Surface Configurations," Journal of Neurophysiology, Vol. 55, No. 6, 1986, pp. 1369-1381.

[3] A. S. Aruin, W. R. Forrest and M. L. Latash, "Anticipatory Postural Adjustments in Conditions of Postural Instability," Electroencephalography and Clinical Neurophysiology/Electromyography and Motor Control, Vol. 109 , No. 4, 1998, pp. 350-359. doi:10.1016/S0924-980X(98)00029-0

[4] A. S. Aruin and M. L. Latash, "Directional Specificity of Postural Muscles in Feed-Forward Postural Reactions during Fast Voluntary Arm Movements," Experimental Brain Research, Vol. 103, No. 2, 1995, pp. 323-332. doi:10.1007/BF00231718

[5] M. Schmid, et al., "Adaptation to Continuous Perturbation of Balance: Progressive Reduction of Postural Muscle Activity with Invariant or Increasing Oscillations of the Center of Mass Depending on Perturbation Frequency and Vision Conditions," Human Movement Science, Vol. 30, No. 2011, pp. 262-278.

[6] J. Massion, "Movement, Posture and Equilibrium: Interaction and Coordination," Progress in Neurobiology, Vol. 38 , No. 1, 1992, pp. 35-56. doi:10.1016/0301-0082(92)90034-C

[7] A. Shumway-Cook and M. Woollacott, "Motor Control: Translating Research into Clinical Practice," 3rd Edition, Lippincott Williams \& Wilkins, Washington DC, 2007,

[8] P. D., Hansen, M. H. Woollacott and B. Debu, "Postural Responses to Changing Task Conditions," Experimental Brain Research, Vol. 73, No. 3, 1988, pp. 627-636. doi:10.1007/BF00406622

[9] K. Van Ooteghem, et al., "Aging Does Not Affect Generalized Postural Motor Learning in Response to Variable Amplitude Oscillations of the Support Surface," Experimental Brain Research, Vol. 204, No. 4, 2010, pp. 505514. doi:10.1007/s00221-010-2316-1

[10] N. Bugnariu and H. Sveistrup, "Age-Related Changes in Postural Responses to Externally- and Self-Triggered Continuous Perturbations," Archives of Gerontology and Geriatrics, Vol. 42, No. 1, 2006, pp. 73-89.

[11] V. Dietz, et al., "Human Stance on a Sinusoidally Translating Platform: Balance Control by Feedforward and Feedback Mechanisms," Experimental Brain Research, Vol. 93, No. 2, 1993, pp. 352-362. doi:10.1007/BF00228405 
[12] J. P. Scholz, et al., "Motor Equivalent Control of the Center of Mass in Response to Support Surface Perturbations," Experimental Brain Research, Vol. 180, No. 3, 2007, pp. 163-179.

[13] TCPS2, "Tri-council Policy Statement: Ethical Conduct for Research Involving Humans," Canadian Institutes of Health Research, Natural Science and Engineering Research Council of Canada, and Social Sciences and $\mathrm{Hu}-$ manities Research Council of Canada, Tri-Council Policy Statement: Ethical Conduct for Research Involving Humans, 2010.

[14] H. Hermens, et al., "Development of Recommendations for SEMG Sensors and Sensor Placement Procedures," Journal of Electromyography and Kinesiology, Vol. 10, No. 5, 2000, pp. 361-375. doi:10.1016/S1050-6411(00)00027-4

[15] J. P. Scholz and G. Schöner, "The Uncontrolled Manifold Concept: Identifying Control Variables for a Functional Task," Experimental Brain Research, Vol. 126, No. 3, 1999, pp. 289-306. doi:10.1007/s002210050738

[16] K. Fujiwara, et al., "Postural Control Adaptability to Floor Oscillation in the Elderly," Journal of Physiological Anthropology, Vol. 26, No. 4, 2007, pp. 485-493. doi:10.2114/jpa2.26.485 